Volume 9, No.3, May - June 2020

International Journal of Advanced Trends in Computer Science and Engineering

Available Online at http://www.warse.org/IJATCSE/static/pdf/file/ijatcse162932020.pdf

https://doi.org/10.30534/ijatcse/2020/162932020

\title{
A Novel approach for Retrieval of Historical Monuments Images using Visual Contents and Unsupervised Machine Learning
}

\author{
Jaimala Jha ${ }^{1}$, Dr. Sarita Singh Bhaduaria ${ }^{2}$ \\ India, jaimala.jha@gmail.com \\ Professor MITS, Gwalior, India, saritamits61@yahoo.co.in
}

\begin{abstract}
In Recent Era monuments image identification and retrieval task is too complex than other dataset images due to its distinct features variation and complex architecture. Structure orientation also plays a vital role for identification and recognition of monuments images. Using content based image retrieval our proposed approach provides a solution of this problem. The aim of proposed work is to make an efficient model to retrieve correct monuments images using machine learning algorithm. Proposed approach extract the handcrafted features by using the RGB Histogram (fast) color features and Histogram of Oriented Gradient texture feature. To make a perfect cluster with minimum outliers of images we used unsupervised machine learning i.e. k-means clustering. This algorithm has been made the retrieval process fast by avoid the whole search for matching similarity in the complete dataset. The experiments show the performance of proposed approach which gives $97 \%$ accuracy for retrieval of monument images.
\end{abstract}

Key words: Monuments image retrieval, RGB Histogram (Fast), Histogram of Gradient, features, k-Means Clustering.

\section{INTRODUCTION}

Monuments image retrieval (MIR) and identification is a novel thought in image retrieval area. Few research studies also explore some methodology regarding monuments recognition and identification. In this regards a novel approach has been proposed for monument image retrieval and identification based on machine learning. Identify the monuments images from the databases according to the user query is a very crucial task nowadays.[1],[2] In this view the demand has come which generate a system i.e. CBIR. Which play an important role to find appropriate content for fine representation. It works on low level features and also deal with high level semantic which is given by the machine. Thus, unsupervised machine learning techniques has a vital role to give the more positive results than others. Here, the proposed approach holds a K-means Clustering technique for image organization as the form of image clusters. This unsupervised clustering does not use labels which define the classes. There is no information presents regarding the category or class label which distinguishes the unsupervised learning concept from supervised learning like, classification, regression etc. Clustering techniques has an elongated and rich history in various fields. The strategy of the clustering dataset which is based upon image content descriptors can resolve the problems that human beings subjectively express an image as essential [4], [6]. Normally, image content descriptors have included two levels features one is low-level features and other is high-level features. Low-level features are described by extracting color, texture and shape features of an image. Further, the high-level feature is based on semantic features that are computed by the human visual observation mechanism, instance, emotion, action, etc [3],[5].

The performance of the system is examined by some parameters i.e. recall, precision and F-measures, that mitigates the semantic gap and hence it will increase the accuracy of the system from the other previous approach [7]. The proposed method is implemented on the Indian Monuments database. It is a synthetic database which contains approx. 1500 images of different categories. The organization of this paper is described as follows: Section 2 discusses the literature survey in this field. Section 3 briefly summarizes the feature extraction techniques that are applied to create the vector dataset. Sections 4 explain the unsupervised $\mathrm{K}$-means clustering techniques that are used to make perfect the clusters. Sections 5 explain the proposed methodology and flow diagram of the system. Section 6 shows the experimental results of the system and Section 7 conclude the benefits and limitations of proposed work with brief the future scope.

\section{RELATED LITERATURE WORK}

This section cover those research studies which is similar to our proposed work like, the techniques and monuments dataset. Few studies have been explored on monuments image retrieval. Padmashree Desai, Jagadeesh Pujari, N.H.Ayachit 
[1], has focused first time in the field of archaeological monuments image retrieval. This work became a foundation line for art lover researcher in this area. This paper contains the idea of automatic classification of monuments images using GLCM and morphological operators and achieved accuracy $90 \%$. The other studies based upon the techniques like machine learning algorithms and various features combination model. Aradhya Saini, Tanu Gupta, Rajat Kumar, Akshay Kumar Gupta,Monika Panwar, Ankush Mittal [2], presents the idea of recognition of monuments images using Deep convolution neural network approach and achieved $92.7 \%$ accuracy of the system. P. Pattanasethanon, B. Attachoo[8], have given a alternative method for image retrieval using two most commonly approach i.e. K-means and Hierachical clustering techniques. This paper emphasis on the problem of CBIR, where outcomes consists of many unrelated feedbacks. Experimental analysis gives the outputs as the form of precision and recall values i.e. 70\% and 50\%. Palepu Pavani T. Sashi Prabha [9] this paper focuses an improved relevance feedback methods with support vector machine (SVM). It decreases the semantic gap between system and user. Also it has given the solution of imbalance training set problem by adding more positive images feedback for user labeling in the system for improving the performance of CBIR system. Neha Mathur, Pankaj Dadheech, Mukesh Kumar Gupta [10] focuses a novel approach which is used two different methods for generating image clusters known as fuzzy edge detection and K-means. This is used to enhance the performance of edge detector and also preserves more significant information about the tumors image. Zubair Khan, Jianjun Ni, Xinnan Fan, Pengfei Shi [11] employing an adaptive histogram-based procedure which requires less user interaction to determine $\mathrm{K}$-means initialization parameters. The results prove that the outcomes of the k-means method are better than other membership-based algorithms of clustering. Ling-Fei Zhang, Chun-Fang Li, Hong-Ru Wang, Min-Yong Shi [12], this proposed work focuses on three classification methods i.e. SVM, Artificial Neural Network and Decision Trees. LBP and HOG are used to extract the pattern and used KIMIA Path960 dataset which contain 960 histopathology images. Out of three methods SVM has given highest accuracy of 90.52\% than other methods. Mohammad Ashraf Ottom , Noor Aldeen Alawad, Khalid M. O. Nahar [13], developed a method which is used for classification of mushroom images. It contains three different algorithms like Support Vector Machines (SVM), K Nearest Neighbors (KNN) and Decision Tree. The illustration results showed $94 \%$ accuracy of the system using KNN technique for best suitable mushroom images. Monika Jain, Dr S. K .Singh [14], presented a unsupervised clustering method known as HDK method, which is used to find unseen patterns for high dimension data form huge amount of dataset. R. Biswas, S. Roy [24], this work emphasis on ROI extraction method used for nodule lungs retrieval in CT images. The proposed method contains features like Region of Interest, Fast discrete curvelet transform (FDCT) and GLCM. Deep Neural Network is used for classification o the images categories. The assessment results had shown the progress in the forms of recall and precision.

\section{FEATURE EXTRACTION TECHNIQUES}

To represent the image in a numerical form and to recognize the image very well for the system there is a need to retrieve the information from the image through visual content like shape, color, and texture. So there are some techniques of color, texture and shape individually and combination of two's like the color moment, HSV histogram, GLCM feature, Tamura technique, SIFT feature, and CEDD, etc. The combinations of proposed features used in this work are discussed below:

\subsection{RGB Histogram (Fast)}

When From the image, this technique extracts the color contents. If the pattern of color is single as compared with the remaining dataset then this RGB histogram acts as an operative representation in terms of color information of an image[15]. From the original RGB histogram, It is four times faster.

An RGB color model contains primary colors R means RED, G means Green and B means Blue colors. Color Histogram can be stated as "Histogram of Three Colors" which means each RGB color channel shows the brightness. From the user input, it takes several bins for each RGB color channel and chooses the value of either un-normalized, L1-normalized or L2-normalized [15]. Suppose if the number of bins is eight then the outcome is calculated as $8 * 8 * 8=512$ for each color channel and achieve a dimension 24 .

\subsection{Histogram of Oriented Gradient (HoG)}

This feature descriptor is used to recognize the human face detection and for object detection. The niche of this feature is to find out the edge or gradient structure of the local shape of the image. Through the categorization of intensity gradients or edge directions, the local edge object and shape within the image is calculated [16]. The histogram of oriented gradients (HOG) is calculated by isolating the image into small linked regions known as cells, then find the histogram of gradients and edge direction of each cell for the pixels inside the cell 24 .

The descriptor is formed by the amalgamation of these histograms. The accuracy of this feature is increased by computing each pixel intensity of an image. This is known as a block. Consequently, the cells which reside in the blocks are normalized by using this value [17]. HOG feature extraction algorithm steps are:

i. Takes color image convert it into a gray image.

ii. Determine each pixel luminance gradients.

iii. For each cell make a histogram of gradient orientations.

iv. Apply Normalization on descriptor blocks to get normalized value.

The luminance gradient is a vector which contain magnitude (m) and orientation $(\theta)$. This basically represented by the change in the luminance [18]. 


\section{MONUMENTS IMAGE RETRIEVAL USING K-MEAN CLUSTERING}

Clustering techniques comes under the category of unsupervised machine learning. It is a basic method for analysis of data. The aim is to partition the large dataset into the number of groups. Each data item in the same group having related characteristics to others, whereas the data items belong to another set having different characteristics [19]. K-Means is mainly targeted on discovering the local minimal solutions depend on the iterative process. Firstly define the clusters numbers (Ck).Every cluster has a mean point known as centroid. Each data points fit in the nearest cluster, and then modified the centroid through their chosen cluster instances and a centroid cannot go outside of the local volume of data. Consequently, the cluster center can move as instances are allocated to it [20]. The major goal of the $\mathrm{k}$-Means clustering algorithm is to provide optimize objective function(f) in spite of the estimation of the distance between the instances and clusters[22. To diminish with-in cluster squared distances, this technique shifts the cluster mean point in the region of space. The position of all instances within the cluster is averaged then recomputed the new centroids value for each clusters. [23] The mathematical interpretation of $\mathrm{K}-\mathrm{Means}$ as our work is:

Initially we have an $\mathrm{mxn}$ vector matrices denoted by $\mathrm{S}$ :

$S=\left(A_{i j}\right) m \times n$ is a matrix of features dataset.

$S=\left\{A_{1}, A_{2}, \ldots, A_{i}, \ldots, A_{m}\right\}$ a data set of $m$ records.

$A m=\left(A_{1 m}, A_{2 m}, \ldots, A_{n m}\right)$ a each record is an n-dimensional vector. Then find the mean of each column of matrix denoted by $\mu \mathrm{i}$

$$
\mu 1=\left[\left(\sum_{i=1}^{m} a_{: .2}\right) / m\right]
$$

Where $i=1,2,3 \ldots \ldots \ldots . n, \mu=\left\{\mu_{1}, \mu_{2}, \mu_{3} \ldots \mu_{n}.\right\}$ then, find the distance which is denoted by $\mathrm{dm}$ :

$$
d_{m}=\left\|A_{m}-\mu\right\|=\sqrt{\sum_{i=1}^{n}\left(A_{i m}-\mu_{i}\right)^{2}}
$$

Based on the minimum Euclidean distance $d_{m}$ of the image feature vectors, the image clusters have been formed. The following sequential steps of k-Means given below:

i. Initially decide no. of clusters (random value of $\mathrm{C}_{\mathrm{n}}$ ) depends on user requirements.

ii. dividing each item into $n$ number of non-empty small groups.

iii. Recognize the mean value of clusters of the recent partition.

iv. Put each points into a particular cluster.

v. Evaluate the distances of each cluster points and allotted points, from centroids distance which is minimum.

vi. After re-assigning the points, again determine centroids of the new clusters produced.

\section{PROPOSED METHODOLOGY}

A central theme of our novel approach is historical monuments images retrieval (MIR) based upon purely CBIR. It works on low level handcrafted features appropriately. The objective of our system to forms a proficient combination of features descriptor for image retrieval. Three terminologies are used in this paper to make the best cluster of the monuments image dataset. Color and shape descriptors are used to extract the prominent features into a numerical value from the images. The significant term is an unsupervised clustering algorithm is applied to generate an efficient cluster with minimum outliers.

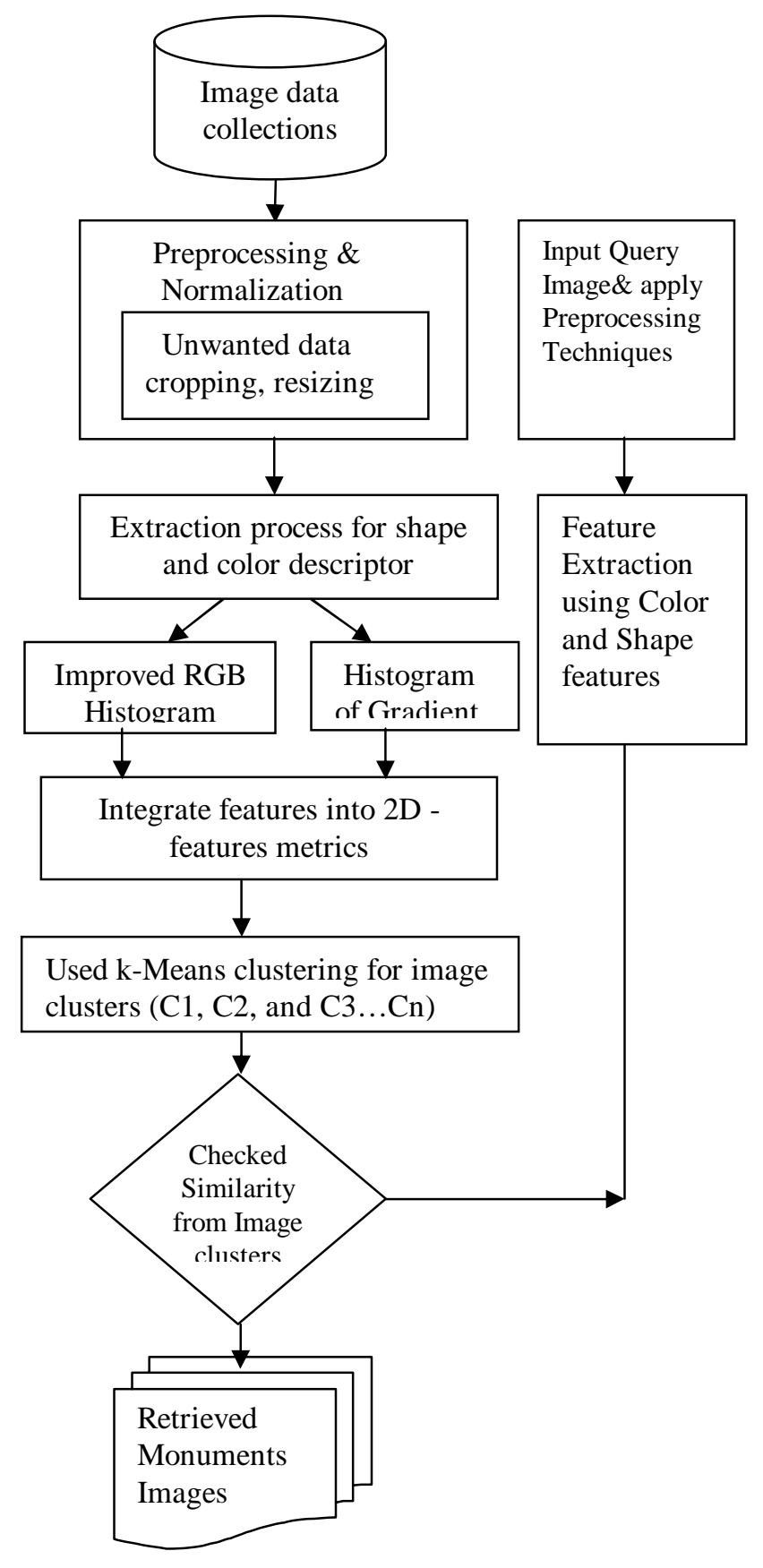

Figure 1: System Flow Diagram 
This methodology contains system flow diagram show in Figure 1. and also mentioned sequential steps of the proposed work.

i. Pick up the folder of monument images repository having images.

ii. Resize the image database i.e. pre-processing for an equal dimension of each image.

iii. Form the feature dataset of images using RGB histogram (fast) and Histogram oriented of gradients descriptors [f1, f2].

iv. Load the formed dataset and assign the number of clusters $\mathrm{k}$ to the k-means clustering algorithm eg. According category wise of database images $(\mathrm{k}=$ $1,2,3, \ldots . . \mathrm{n})$.

v. Consequently, we attained the clustered group of images.

\section{EXPERIMENTAL ANALYSIS AND OUTCOMES}

For experimental illustration of the proposed work the Matlab version (2017) and 64 bit operating system is used. For image acquisition from different places used Motorola (Moto $E^{3}$ power) cell phone. Its android version is 6.0. System has contained dataset of 10 classes with different categories of the monument's image. Figure 2 shows synthetic database which contain different classes of Indian monuments images. This database contains 1000 images of ten classes. Images present in a dataset are collected manually visiting the archaeological monuments individually and then Pre-processing is applied to make a dataset suitable for research purposes. For preprocessing apply cropping function for removing unwanted objects reside in the images. Then apply a resize function to provide the same dimension.Monuments images dataset is available on below link.

\section{https://drive.google.com/open?id=12e74Ozi_RxOMalT_LE1 I35SOOIFXnRGD}

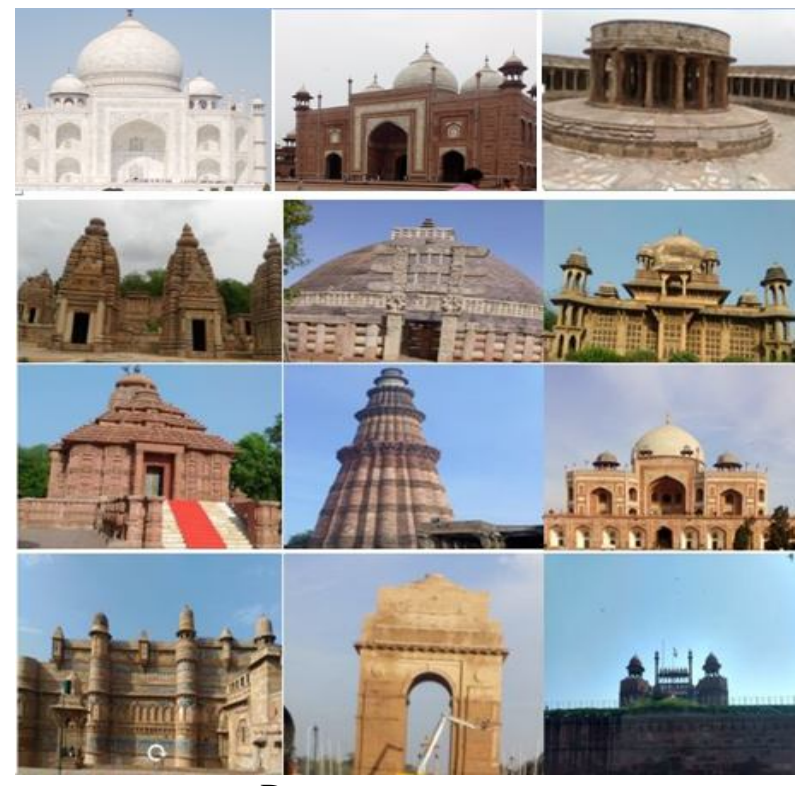

Figure 2: Dataset of Historical Monuments

\subsection{Performance Measures}

The effectiveness of the system is measured by Precision, Recall, and F-Measure. Precision is calculated as how much relevant images retrieved of a particular category. The Recall is calculated as the total number of relevant images present in a particular cluster and F-Measure tells us about the accuracy of the system [7].

$$
\begin{aligned}
\text { Precision }(\mathrm{p}) & =\frac{\text { No. of relevant images retrieved }\left(\mathrm{N}_{\mathrm{r}}\right)}{\text { Total number of images retrieved }\left(\mathrm{N}_{\mathrm{t}}\right)} \\
\text { Recall }(\mathrm{r})= & \frac{\text { No. of relevant images retrieved }\left(\mathrm{N}_{\mathrm{r}}\right)}{\text { Total no. of relevant images in database }\left(\mathrm{N}_{\mathrm{d}}\right)} \\
\text { F-Measure } & =\frac{2 * \text { Precision } * \text { recall }}{\text { Precision }+ \text { recall }}
\end{aligned}
$$

\subsection{Retrieved Output as Image Clusters}

After applying the k-means clustering algorithm the monuments image cluster is produces as the form of output with cluster indices. Cluster indices give the cluster numbers for the image, which means that each image belongs to a particular cluster. The following Figure 3 shows the output of the cluster image category wise. This is the sample result for few class categories. In Figure 3 cluster 1 shows the Sanchi Stupa class, cluster 2 shows the Redfort class and third cluster shows the Qutub Minar class. In this experimental analysis, clusters contain some outliers. The outlier is an image that does not belong to that class. But the percentages of outliers are very less in this system. Table 3 depicts the precision and recall values for each category of images exist in the database. These two parameters give the performance of the system.

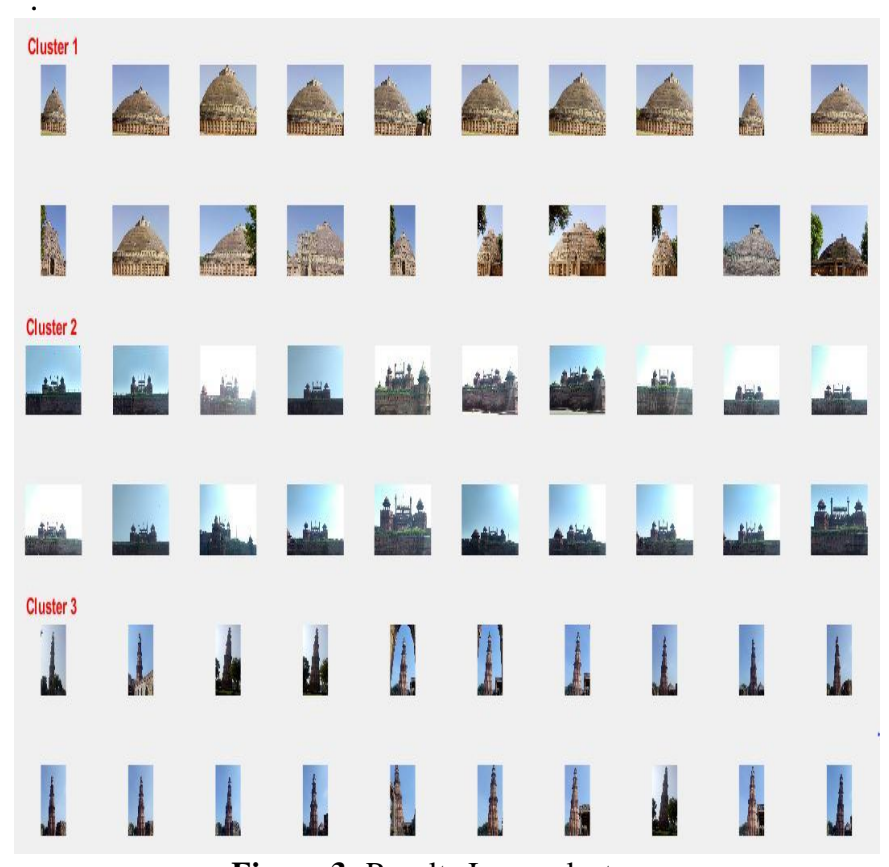

Figure 3: Results Image clusters 
Based on the clusters output it is easy to retrieve the similar query image from the image clusters. This approach presents the concept of reduced amount of time for query matching process execution. The whole image set is divided into chunks of clusters, so that the searching will be done based on the best suitable cluster.

Table 1: Calculated precision (\%) based on similarity metrics

\begin{tabular}{|l|c|c|l|}
\hline $\begin{array}{c}\text { Image } \\
\text { category }\end{array}$ & $\begin{array}{l}\text { Euclidea } \\
\text { n }\end{array}$ & $\begin{array}{l}\text { Normalized } \\
\text { Euclidean }\end{array}$ & $\begin{array}{c}\text { City } \\
\text { block }\end{array}$ \\
\hline Category 1 & 99.66 & 94.66 & 96.00 \\
\hline Category 2 & 98.11 & 97.33 & 86.00 \\
\hline Category 3 & 98.67 & 90.33 & 89.33 \\
\hline Category 4 & 98.45 & 87.57 & 89.33 \\
\hline Category 5 & 99.00 & 97.66 & 92.66 \\
\hline Category 6 & 97.67 & 94.12 & 92.66 \\
\hline Category 7 & 97.89 & 96.66 & 97.00 \\
\hline Category 8 & 96.14 & 90.33 & 89.33 \\
\hline Category 9 & 97.24 & 94.33 & 90.33 \\
\hline Category 10 & 98.00 & 90.66 & 87.00 \\
\hline Avg. & 98.083 & 93.365 & 90.731 \\
\hline
\end{tabular}

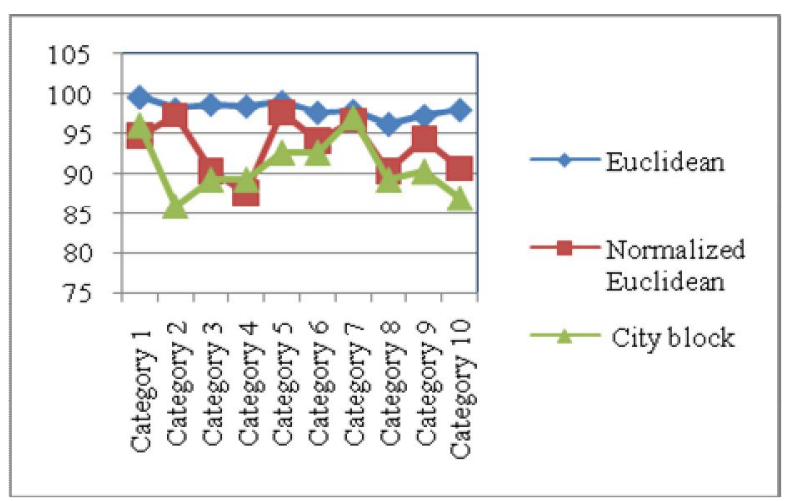

Figure 4: Precision Graph based on similarity metrics

Table 2: calculated Recall (\%) based on similarities metrics

\begin{tabular}{|l|l|l|l|l|l|l|}
\hline \multicolumn{4}{|c|}{ Existing approach [21] } & \multicolumn{3}{c|}{ Proposed approach } \\
\hline sno & Precision & Recall & $\begin{array}{l}\text { F- } \\
\text { scores }\end{array}$ & Precision & Recall & $\begin{array}{l}\text { F- } \\
\text { scores }\end{array}$ \\
\hline 1 & 94.66 & 78.56 & 85.86 & 99.66 & 95.65 & 97.6 \\
\hline 2 & 87.00 & 72.21 & 78.91 & 98.11 & 97.56 & 97.04 \\
\hline 3 & 90.66 & 75.19 & 82.20 & 98.67 & 95.78 & 97.20 \\
\hline 4 & 76.66 & 63.62 & 70.42 & 98.45 & 93.62 & 96.80 \\
\hline 5 & 98.66 & 81.34 & 89.16 & 99.00 & 95.66 & 97.85 \\
\hline 6 & 75.66 & 62.79 & 68.62 & 97.67 & 94.78 & 96.20 \\
\hline avg & $\mathbf{8 7 . 2 1}$ & $\mathbf{7 2 . 2 8}$ & $\mathbf{7 9 . 1 9}$ & $\mathbf{9 8 . 5 9}$ & $\mathbf{9 5 . 5 0}$ & $\mathbf{9 7 . 1 1}$ \\
\hline
\end{tabular}

Table 1 shows the calculate values of precision from different similarities metrics which gives the better performance of Euclidean distance similarity than other metrics. Table 2 also contains recall values. Here we also found same outcomes as above said. The Euclidean metrics generate best results for our proposed approach for both cases precision as well as recall.

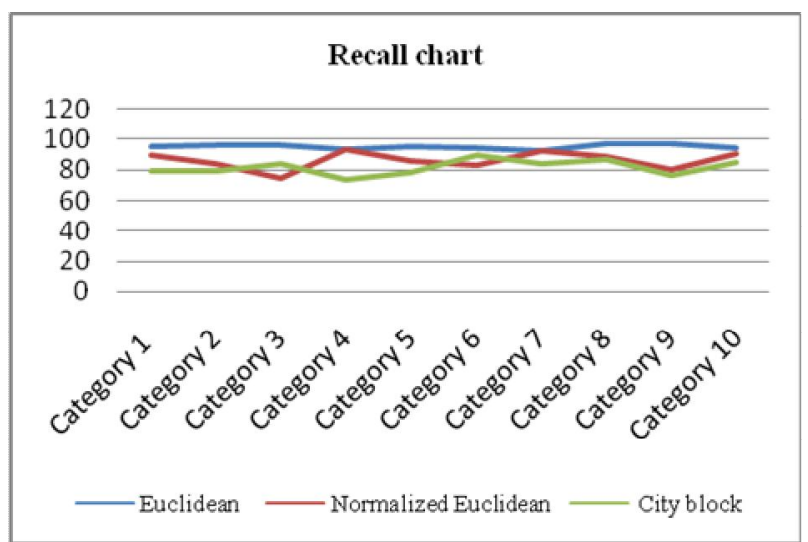

Figure 5: Recall Graph based on similarity metrics

Table 3: Assessment of Proposed Methodology

\begin{tabular}{|c|l|l|l|}
\hline $\begin{array}{c}\text { Image } \\
\text { category }\end{array}$ & Euclidean & $\begin{array}{c}\text { Normalized } \\
\text { Euclidean }\end{array}$ & $\begin{array}{c}\text { City } \\
\text { block }\end{array}$ \\
\hline Category 1 & 95.6 & 89.56 & 79.68 \\
\hline Category 2 & 96.00 & 83.78 & 79.72 \\
\hline Category 3 & 95.78 & 74.94 & 84.11 \\
\hline Category 4 & 93.62 & 93.19 & 74.11 \\
\hline Category 5 & 95.00 & 85.88 & 78.56 \\
\hline Category 6 & 94.78 & 83.11 & 89.76 \\
\hline Category 7 & 92.66 & 92.11 & 83.99 \\
\hline Category 8 & 97.00 & 88.56 & 86.68 \\
\hline Category 9 & 96.67 & 80.85 & 76.82 \\
\hline Category 10 & 94.77 & 90.29 & 84.77 \\
\hline Avg. & $\mathbf{9 5 . 1 8}$ & $\mathbf{8 6 . 2 2}$ & $\mathbf{8 1 . 8 2}$ \\
\hline
\end{tabular}

For evaluating the accuracy of our proposed method considers the precision and recall values based on Euclidean distance metrics and calculated F- score also which shows in Table 3. We compare these outcomes with related existing work i.e. [21] and current proposed work based on F-measures parameter. It is the accuracy agent which provides the performance index. We consider only six categories of images out of ten categories. Based on the results of Table 1 and Table 2 the precision and recall graph have been plotted showed in Figure 4 and Figure 5. These figures contain precision and recall values of all ten categories of images. The Table 3 depicts the information regarding the performance of the proposed method as the form of precision, recall and F-scores. Overall illustrations conclude that our methodology obtain better outcomes than other recent works in this area. Figure 6 shows the accuracy chart in the form of graph. This graph contains all three central performance parameters for different images categories from the dataset. 


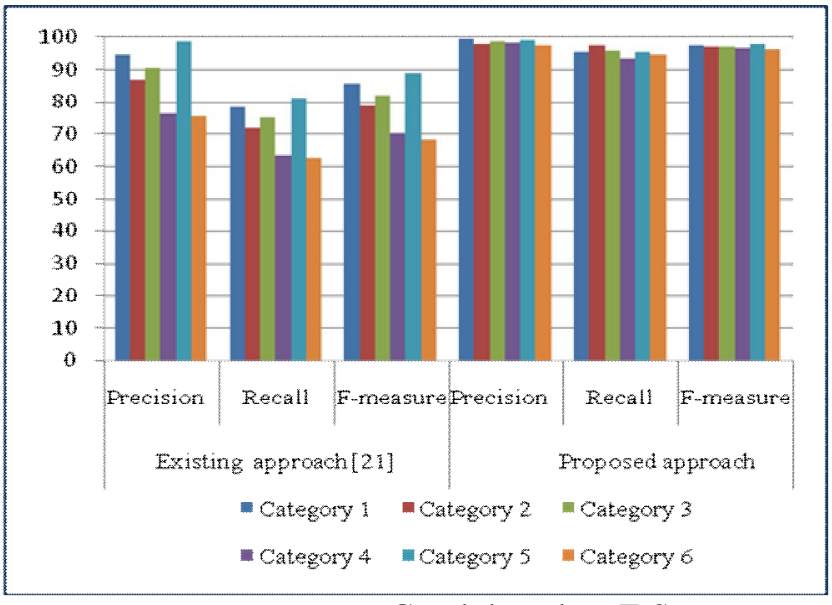

Figure 6: Accuracy Graph based on F-Score

\section{CONCLUSION WITH FUTURE SCOPE}

The proposed new approach offers a framework for monuments image retrieval (MIR). The scope of this research is focused on reduced the semantic gap of features vectors based upon the features extraction techniques. This technique supply only 1081 handcrafted features set of the input images. This small size features vectors generate finest clusters with minimum outliers according to the category of the image database. Proposed method has achieved $97.11 \%$ accuracy in terms of F-scores. For further improvements used, suitable preprocessing techniques and more appropriate handcrafted features that will reduce the semantic gaps of the image contents and provide desired information according to images. Other researcher can also used Deep learning in-place of machine learning techniques which will help for better classification of historical monuments images.

\section{REFERENCES}

1. Padmashree Desai, Jagadeesh Pujari, N.H.Ayachit. Classification of Archaeological Monuments for Different Art forms with an Application to CBIR, IEEE International Conference on Advances in Computing, communications and Informatics (ICACCI), IEEE, pp. no. 1108-1112, 2013.

2. Aradhya Saini, Tanu Gupta, Rajat Kumar, Akshay Kumar Gupta,Monika Panwar, Ankush Mittal. Image based Indian Monument Recognition using Convoluted Neural Networks, IEEE International Conference on Big Data, IoT and Data Science (BID) Vishwakarma Institute of Technology, Pune, pp.no. 138-142, Dec 20-22, 2017.

https://doi.org/10.1109/BID.2017.8336587

3. Afshan Latif, Aqsa Rasheed, Umer Sajid, Jameel Ahmed, Nouman Ali, Naeem Iqbal Ratyal, Bushra Zafar, Saadat Hanif Dar,Muhammad Sajid, Tehmina Khalil. Content-Based Images Retrieval and Features Extraction: A Comprehensive Reviews,
Mathematical Problems in Engineering Volumes, HINDAWI, pp. no.1-21, 2019. https://doi.org/10.1155/2019/9658350

4. S Mahajan, D. Patil. Image Retrieval Using Contribution-based Clustering Algorithm with Different Features Extraction Techniques, International Conference on IT in Business, Industry and Government (CSIBIG) IEEE, 2014.

5. N A. K. Jain. Data clustering: 50 years beyond K-means, Pattern Recognition. Letter, vol. 31, no. 8, pp. 651-666, 2010.

6. Mussarat Yasmin, Sajjad Mohsin, Muhammad Sharif. Intelligent Image Retrieval Techniques: A Survey, journal of Applied Research and Technology Vol.12, pp.no 87-103. February 2014. https://doi.org/10.1016/S1665-6423(14)71609-8

7.R. Jayaswal, J. Jha, and R. Devesh. An Effective Method of Image Mining using K-Medoid Clustering Technique, International Journal of Computer Science Engineering Open Access Res., no. 56, pp. 206-214, 2017.

8. P.Pattanasethanon1 B. Attachoo. An alternative approach for unsupervised cluster based image retrieval International Journal of Physical Sciences Vol. 7(39), pp. 5498-5510, ISSN 1992 - 1950, 16 October, 2012.

9. Palepu Pavani ,T. Sashi Prabha. Content Based Images Retrieval using Machine Learning Approach, Advances in Intelligent Systems and Computing Springer. 247, 173, Springer International Publishing Switzerland, 2014.

10. Neha Mathur, Pankaj Dadheech, Mukesh Kumar. K-means Clustering Based Fuzzy Edge Detection Technique On MRI Images, International Conference on Advances in Computing and Communications, IEEE pp. 330-333, 2015. https://doi.org/10.1109/ICACC.2015.103

11. Zubair Khan, Jianjun Ni, Xinnan Fan, Pengfei Shi. An Improved KMeans Clustering Algorithms Based on an Adaptive Initial Parameter Estimation Procedure for Image Segmentation. International journal of innovating computing information and control vol.13 no.5,pp no. 1509-1525, ISSN 1349-4198, 2017.

12. Lingling Zhang, Minnan Luo, Jun Liu, Zhihui Li and Qinghua Zheng. Diverse Fuzzy C-Means for Image Clustering, International Journal of Patterns recognition letters, Elsevier 2018.

13. Mohammad Ashraf Ottom, Noor Aldeen Alawad, Khalid M. O. Nahar.Classification of Mushroom Fungi using Machine Learning Techniques, International Journal of Advanced Trends in Computer Science and Engineering Volume 8, No.5, October 2019 pp.2378-2385. https://doi.org/10.30534/ijatcse/2019/78852019

14. Monika Jain, Dr S.K. Singh. An Efficient Content Based Image Retrieval Algorithm using 
clustering Techniques for Large Dataset, IEEE 4th International Conference on Computing Communication and Automation (ICCCA) IEEE 2018.

15. Sonika Jindal, Sunpreet Kaur. Image Retrievals using Variants of ColorHistogram Techniques, International Journal of Advanced Research in Computer Science and Software Engineering Volume 6, Issue 10, ISSN: 2277 128X, October 2016.

16. N. Dalal and B. Triggs. Histograms of oriented gradients for human detection, IEEE Computer Society Conference on Computer Vision and Pattern Recognition (CVPR'05), vol. 1, pp. 886-893,2005.

17. N. Dalal, B. Triggs, C. Science. Histogram of oriented gradients, Computer. Vision, Pattern Recognition, and Neural. Network. Classifier, pp. 1-6, 2016.

18. Taha J. Alhindi, Shivam Kalra, Ka Hin Ng, Anika Afrin, Hamid R. Tizhoosh. Comparing LBP, HOG and Deep Features for Classification of Histopathology Images, IEEE World Congress on Computation Intelligence, July 2018.

19. V. K. Dehariya, S. K. Shrivastava, R. C. Jain. Clustering of Image Data Set Using K-Means and Fuzzy K-Means Algorithms proceeding International conference on CICN, IEEE pp. 386391, 2010.

https://doi.org/10.1109/CICN.2010.80

20. Shubhi Misra, Atul Kumar Verma. Content Based Image Retrieval Using K-Means Algorithm International Journal of Applied Engineering Research ISSN 0973-4562 Volume 13, Number 7 pp. 5562-5564. 2018.

21. Ravi Devesh,J. Jha. An Efficient Approach for Monuments Image Retrieval Using Multi-visual Descriptors. Proceeding of the Second International Conference on Microelectronics, Computing \& Communication Systems (MCCS 2017), Lecture Notes in Electrical Engineering 476, Springer Nature Singapore. 2019. https://doi.org/10.1007/978-981-10-8234-4_25

22. P.Kumar Guru Diderot, N Vasudevan, k sakthidasan. An efficient fuzzy C-means clustering based image dissection algorithm for satellite images International Conferences of communication and signal processing, 2019, India. https://doi.org/10.1109/ICCSP.2019.8698054

23. Romsa Dhmeja, J. Jha. Analysis of Clustering Methods used in CBIR System, International Journal of Innovative Research in Science, Engineering and Technology vol. 9, issue 4, April 2020.

24. R. Biswas, S. Roy, Content Based CT Image Sign Retrieval using Fast Discrete Curvelet Transform and Deep Learning. International Journal of Advanced Trends in Computer Science and Engineering, pp. 854 - 863 Volume 8, No.3, June 2019, ISSN 2278-3091.

https://doi.org/10.30534/ijatcse/2019/80832019 